\section{Effects of US intensity on heart rate in delay conditioning and pseudoconditioning ${ }^{1}$}

WIIIIIAH F. CAUL, ROBERT E. MILLLER, and JAMLS H. BANKS, JR..? Laboratory of Clinical Science. School of Medicine, University of Pittsburgh, Pittsburgh, Pa. I5213

Heart rates of rats were obtained during seven aversive conditioning sessions and four extinction sessions. Two levels of shock intensity were used. The magnitude of the cardiac response to the $C S$ was greater under high shock than under low shock. In the second experiment, the two shock levels were administered to two groups of rats in a pseudoconditioning procedure that specifically precluded CS-US pairing. The cardiac response to the CS in these two groups was indistinguishable from that of the comparable conditioning groups.

The presumption that responses mediated via the autonomic nervous system should reflect the onset and cessation of conditioning stimuli that have been associated with noxious events has generated a great deal of investigation. In the case of some systems, notably the cardiovascular, however, it is clear that there is considerable question concerning the nature of the conditioned response. Thus, for example, various investigators have reported decelerative heart-rate changes during conditioning (e.g., Holdstock \& Schwartzbaum, 1965; Fitzgerald \& Vardaris, 1966), while others have found accelerative changes in rate (e.g., Miller, Banks, \& Caul, 1967; Overmier, 1966). While it is true that these experiments differ from each other in terms of apparatus, Ss, and procedure, it has not proven easy to understand the vagaries of conditioned cardiac rate responses.

The present investigations were performed to examine some basic parameters in aversive conditioning. The initial experiment was designed to assess the effect of different shock intensities on heart rate during classical conditioning.

\section{EXPERIMENT 1}

$$
\text { Method }
$$

Sixteen male Carworth CFN rats, ranging in weight from 217 to $252 \mathrm{~g}$ at the beginning of the experiment, served as Ss. The conditioning apparatus was one chamber of a shuttlebox and was

Fig. 1. Tachograph data for Groups $\mathbf{C H}$ and $\mathrm{CL}$ for the seven conditioning days.
$8 \times 12.5 \times 8$ in. Shock was delivered to the grid floor through a Foringer scrambler. Heart rate was recorded from a clip around the thorax of the rat that held two dime-sized electrodes to the shaved sides of the animal.

\section{Procedure}

Animals were housed in groups of eigh and were given food and water ad lib. Each animal was handled for $3 \mathrm{~min}$ a day for 5 days. On each of the next 2 days, all Ss were given a 10 -min adaptation period in the apparatus with electrodes attached. On the 3 rd and tinal day of adaptation, each rat received 10 trials of the buzzer (CS) alone. The CS increased the sound-pressure level to $68 \mathrm{~dB}$ from the background level of $60 \mathrm{~dB}$. Beginning with the $3 \mathrm{rd}$ day of adaptation and continuing through 7 conditioning and 4 extinction days, the procedure was as follows: A rat was put in the apparatus after the heart-rate clip was attached and electrode paste inserted between the electrodes and the skin. After $2.5 \mathrm{~min}, 10$ trials were given at intervals of $1.5 \mathrm{~min}$. EKG and tachograph records were taken continuously. Two minutes after the 10th trial, the animal was removed from the box, and the thorax was washed to remove the electrode paste.

For eight rats (Group $\mathrm{CH}$ ), the US intensity was $2.0 \mathrm{~mA}$, while for eight other rats (Group CL), the US intensity was $0.6 \mathrm{~mA}$, as measured through a $10 \mathrm{~K}$ ohm resistor. The CS lasted $6.5 \mathrm{sec}$ and terminated at the end of the $0.5-\mathrm{sec}$ scrambled shock. Extinction was identical to conditioning except that shock was never presented.
During the adaptation days as well as during conditioning and extinction days, continuous tachograph records were taken for each session. Starting $5.5 \mathrm{sec}$ prior to onset of the CS, the tachograph record was sampled every second for 25 samples and then every $5 \mathrm{sec}$ for 12 more samples. Trials $1,3,5,7$, and 9 were sampled each day. Although this type of analysis does not deal with adjacent $R \cdot R$ intervals, it was hoped that any biphasic response to the CS might be revealed.

\section{Results}

Adaptation (3rd day). The analyses of heart-rate responses to the CS were based on the means of the six tachograph samples taken at 1-sec intervals immediately preceding a trial and the means of six similar samples taken during the 6-sec CS. The intertrial heart-rate level decreased over trials for all 16 animals $(\mathrm{p}<.05)$, and the direction of the response to the CS changed from a nonsignificant decrease in rate on Trial 1 to a significant increase in rate on subsequent trials ( $\mathrm{p}<.01$ ).

Conditioning and extinction. The tachograph data pooled over the 7 conditioning days are presented in Fig. 1. Inspection of this figure, as well as the individual tachograph records, suggests that the response to the CS was monotonic and not biphasic for both groups. Because of this result and the finding that the heart-rate acceleration developed over trials in a highly similar way within each day, further analyses were based on the means of heart-rate samples taken during the 6-sec pre-CS period and the 6-sec CS interval.

The top plot of Fig. 2 shows these data for the two groups for the third adaptation day, as well as the conditioning and extinction days. During conditioning, the acceleration of heart rate to the CS was reliable for both groups $(p<.01)$ and $\mathrm{did}$ not change in magnitude over days. The

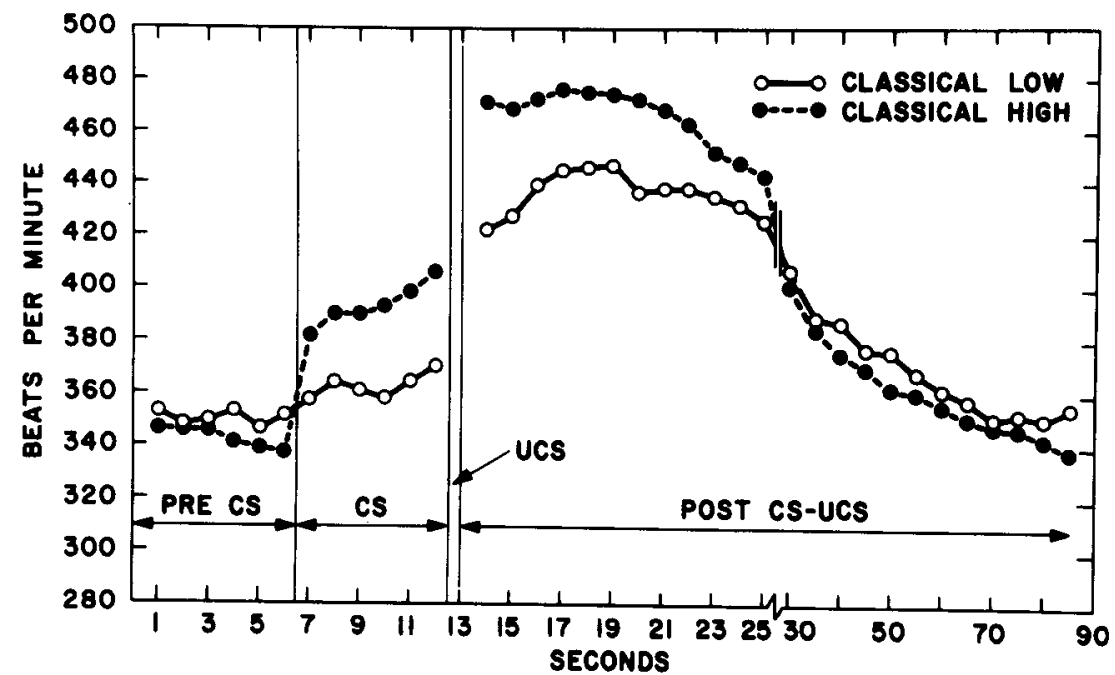


Fig. 2. Experiments 1 and 2. Mean pre-CS and CS heart rate as a function of days.

magnitude of the response to the CS, however, was a function of US intensity. The high shock produced a greater tachycardia than did the low shock $(\mathrm{p}<.01)$.

During extinction, Group $\mathrm{CH}$ continued to show a tachycardia to the $\operatorname{CS}(\mathrm{p}<.01)$, the magnitude decreasing over days $(p<.05)$, while for Group CL the response to the CS extinguished rapidly, resulting in a nonsignificant difference between pre-CS and CS periods over the 4 extinction days.

\section{EXPERIMENT 2}

Experiment 2 investigated heart rate as a function of US intensity measured during pseudoconditioning. This control procedure was similar to that used by Holdstock \& Schwartzbaum (1965) in establishing that the bradycardiac response in their study was dependent on CS-US pairings.

\section{Method}

The Ss were 16 male Carworth CFN rats, ranging from 227 to $263 \mathrm{~g}$ at the beginning of the experiment. Eight animals were assigned to the pseudoconditioning high-shock group (PH), and eight were assigned to the pseudoconditioning low-shock group (PL). The only change in conditioning procedures from that used in Experiment 1 consisted of introducing a variable interval between the termination of the CS and the onset of the US. On each trial, an interval of $22,26,30$, or $34 \mathrm{sec}$ was assigned randomly. Since analyses of the tachograph data of Experiment 1 had revealed a monotonic response to the $\mathrm{CS}$, the unit of analysis during conditioning and extinction of Experiment 2 was the sum of heart beats during the 6-sec pre-CS and 6-sec CS period of each trial.

\section{Results}

Adaptation. During the third adaptation day, which consisted of 10 presentations of the $\mathrm{CS}$, the pre-CS heart rate decreased $(p<.01)$ and there was a significant increase in rate to the stimulus $(p \leqslant .01)$ for both groups of animals.

Conditioning and extinction. The bottom plot of Fig. 2 shows the data for the pseudoconditioning groups during the third adaptation day, pseudoconditioning, and extinction. The acceleration in heart rate to $C S$ presentation was reliable for both groups $(p<.01)$ and was a function of shock intensity $(p<.01)$. The magnitude of the tachycardia to the CS increased over days for Group PL $(p<.05)$. During extinction, both groups continued to show an increase in rate to the CS $(p<.01)$, while the magnitude of

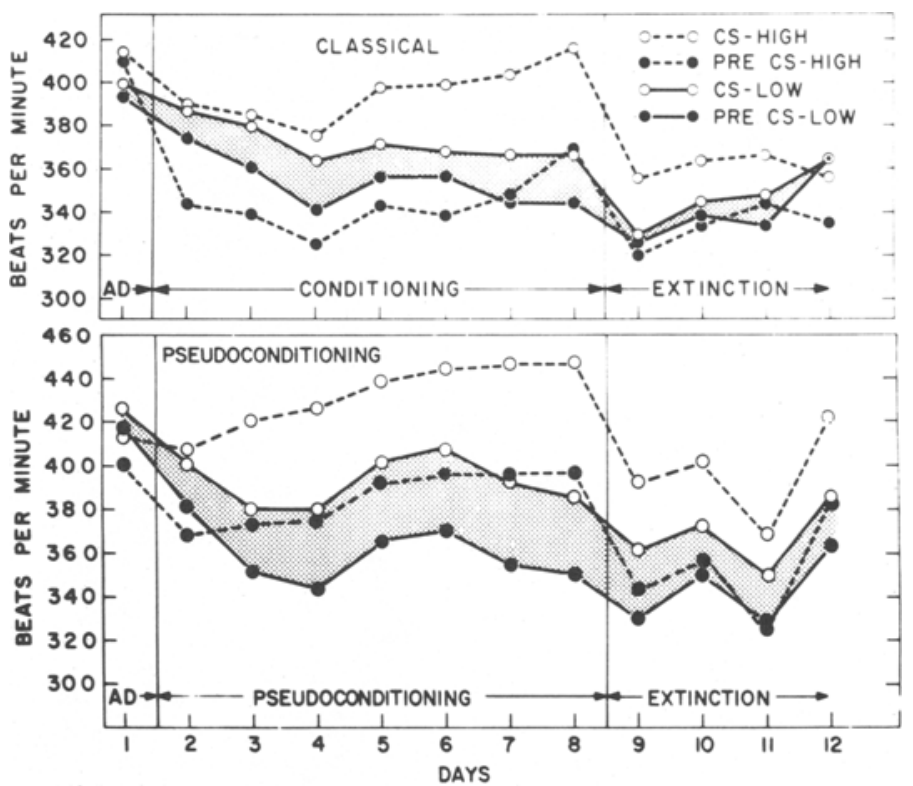

this response decreased over days only for Group PL $(\mathrm{p}<.05)$.

\section{DISCUSSION}

Although few studies measuring heart rate have been concerned with US intensity, it has been reported that both cardiac acceleration during shock and deceleration after shock are monotonic increasing functions of shock intensity (Church, LoLordo, Overmier, Solomon, \& Turner, 1966). In contrast to the present results, Holdstock \& Schwartzbaum (1965) as well as Fitzgerald \& Vardaris (1966) report bradycardiac responses. This discrepancy possibly is attributable to a major procedural difference in that their Ss were continuously restrained throughout testing and had extremely high intertrial heart rates.

Although motor activity was not recorded for Groups $\mathrm{CL}$ and $\mathrm{CH}$, the categories of moving, standing, and grooming were recorded during pre-CS and CS intervals for Groups PL and PH. During the first three conditioning sessions, there was essentially no activity. All animals displayed the typical freezing response to the situation. The heart-rate response during these days, however, was dependent on shock intensity. On the 4th conditioning day, both groups showed increased activity during the $\mathrm{CS}$, while pre-CS activity remained at minimal levels. During the last 4 conditioning days, the average number of trials during which pre-CS activity occurred was 0 for Group PL and 0.12 for Group PH. During the CS, Group PL showed some activity on an average of 2.78 trials and Group PH on an average of 4.60 trials. This increased activity to the CS during the last 4 conditioning days, however, is not apparent in the heart-rate data for these days. It seems, therefore, that just as pre-CS rates can change independently from activity (Caul \& Miller, 1968), cardiac response to a stimulus does not necessarily reflect the level of somatomotor activity.

The results of Experiment 2 pose a major question. What are the parameters that determine cardiac acceleration in conditioning situations? From the similarity of the data for Groups PL and $\mathrm{CL}$ as well as for Groups $\mathrm{PH}$ and $\mathrm{CH}$, it is apparent that the heart-rate response to the CS observed in Experiment 1 was not dependent on CS-US pairings. It has been pointed out (Holdstock \& Schwartzbaum, 1965) that few studies of cardiac conditioning have employed rigorous controls for pseudoconditioning. The present results emphasize the need for such controls before heart-rate responses to a stimulus can be considered conditioned responses. The finding that the acceleratory response under pseudoconditioning procedures was a function of US intensity, as it was under the classical delay-conditioning procedures, appears to fit under the rubric of sensitization. The questions of conditioning vs pseudoconditioning vs sensitization have proven to be issues with regard to several response systems (e.g., Jacobson, Horowitz, \& Fried, 1967; Kimble, 1961) and appear highly relevant to the area of cardiac conditioning. REFERENCES

CAUL, W. F., \& MILLER, R. E. Effects of shock probability on heart rate of rats during classical conditioning. Physiology \& Behavior, 1968, 3, 865-869.

CHURCH, R. M., LOLORDO, V., OVERMIER, J. B., SOLOMON, R. L., \& TURNER, L. H. 
Cardiac response to shock in curarized dogs: 1.fiects of shock intensity and duration. warnine vinal. and prior experience with shock. Journal of Comparative \& Phy siological Pyithology. 1966,62,1-7.

IITZ(;FRALI), R, D.. \& VARDARIS, R. M. Classical conditioning of heart rate deceleration in the rat with continuous and partial reinforcement. Pyychonomic Science. $1966,6,437-438$

HOLDSTOCK. T. L.. \& SCHWARTZBALM, J. S. Classical conditioning of heart rate and salvanic skin response in the rat. Psychophysiology. 1965, 2, 25-38.

JACOBSON. A. L. HOROWTTZ, S. D., \& I RIFD. C. Classical conditioning, pseudoconditioning. or sensitization in the planarian. Journal of Comparative \& Physiological Psychology, 1967, 64, 73-79.

KIMBLI: $\mathrm{G}_{\text {. A. Hilgard and Marquis }}$
Conditioning and learning. New York Appleton-Century-Crofts. 1961. Pp. 59-65.

WILLER, R. I... BANKS. J. H.. JP.. \& CACL. IV. 1. Cardiac conditioned responses in avoidance and yoked-control rats. Psychonomic Science. 1967.9.581-582.

OVERMIER, 3. B. Instrumental and cardiac indices of Pavlovian fear conditioning as a function of US duration. Joumal of Comparative \& Physiological Psychology. $1966,62,15-20$.

\section{NOTES}

1. This investigation was supported in part by a research grant (IM-487 C-15) from the National Institute of Mental Health of the National Institutes of Health, United States Public Health Service, and in part by the Commonwealth of Pennsylvania.

2. Now at U.S. Army Behaviorał Science Research Laboratory, Washington. D.C.

\section{Further study of "learned" activity in food-deprived rats}

\author{
TOMI S. ALLISON, ${ }^{2.3}$ Washington State \\ University, Pullman, Wash. 99163
}

Twenty rats were maintained on a 21-h food-deprivation schedule in activity wheels for 35 days. Ten Ss had freedom to run right up to feeding time daily and 10 were blocked out of the wheels during the last hour before feeding to reduce any reinforcing effect of feeding on running. Wheel running increased significantly from the third to second hours before feeding in both groups, but there was no significant difference between groups. Food presentation was observed to be contingent upon being in side cages rather than upon running in $S s$ fed immediately after opportunity to run. The findings did not support the hypothesis of Reid \& Finger (19.55) that increased wheel running prior to feeding is a function of reinforcement of running by subsequent feeding.

Activity-wheel running in food-deprived rats increases markedly as feeding time approaches (Reid \& Finger, 1955). The hypothesis that this phenomenon results from reinforcement of running due to temporal proximity to feeding (Reid \& Finger, 1955) has been strengthened by reports that rats that are fed immediately after their last opportunity for running run more than rats that are blocked out of the wheel for $1 \mathrm{~h}$ before feeding (Finger, Reid. \& Weasner, 1957; Hall, 1958). The present experiment was undertaken to test this hypothesis further.

One problem with the experiments by Finger et al (1957) and Hall (1958) is that the investigators compared wheel running for different hours in their groups. Running during the last hour before feeding in immediately fed $S s$ was compared with running during the hour beginning $2 \mathrm{~h}$ before feeding in Ss that were blocked from the wheels during the last hour before feeding. Therefore, it is not clear if the differences between groups were a function of differences in temporal proximity of running to feeding or the difference in temporal proximity of the comparison hours to feeding.

In the present experiment, wheel running during the second and third hours before feeding was compared between Ss fed immediately after opportunity for running and Ss blocked from the wheels for $1 \mathrm{~h}$ before feeding. Thus, the two comparison hours (second and third before feeding) were the same for both groups, but one group could run during the last hour before feeding and one group could not run. In addition, the relationship between running and events at feeding time was analyzed for possible reinforcing contingencies.

\section{SUBJECTS AND APPARATUS}

Twenty male hooded rats, 110 days old at the start of habituation, from the colony at Washington State University, were housed throughout the experiment in standard Wahmann activity wheels equated for sliding torque by the method of Lacey (1944) and equipped with cardboard side panels to reduce interaction among Ss. Wheel revolutions were recorded from outside the experimental room by magnetic counters and a 20-pen event recorder. A 12-h light cycle was maintained by an automatic timer. Room temperature was maintained at $72^{\circ} \mathrm{F}$.

\section{PROCEDURE}

The Ss were first given a 7-day habituation period in the wheels under ad lib food and water conditions. A 21-h food-deprivation schedule, with water continuously available, was begun after the 7 th day of habitaution and continued for 35 days. The $E$ entered the experimental room daily at the start of the last hour before feeding to block the delayed-feeding (DF) Ss out of the wheels and to check the immediate-feeding (IF) Ss. A second entry occurred at the end of the hour to feed Ss in both groups. Group DF Ss were again given access to the wheels after food was placed in the side cages. Remaining food was removed from the side cages after $3 \mathrm{~h}$ of feeding, and water supplies were replenished. The two groups of $10 \mathrm{Ss}$ each were run in succession due to equipment and facility limitations, but care was taken to equate extraneous conditions as closely as possible between groups.

Data were collected daily throughout the deprivation period. Magnetic counter readings were recorded for the $3 \mathrm{rd}$ and $2 \mathrm{nd}$ hours before feeding. Event recordings were obtained daily for the 2 nd hour before feeding in both groups and for the last hour before feeding in Group IF.

RESULTS AND DISCUSSION

The mean number of wheel revolutions, for blocks of 5 days, from the second (Hour 2) and third (Hour 3) hours before feeding are shown in Fig. 1. Only the data from the last block of 5 days were analyzed since concern was with the final level of activity produced by deprivation training. Groups by Hours interaction was not significant $(F<1)$. The difference in amount of running between the second and third hours before feeding was significant $(F=17.63, \mathrm{df}=1 / 18, \mathrm{p}<.01)$, indicating that deprivation training did produce a significant increase in running during Hour 2. However, the difference between groups was not significant $(F<1)$ and, in fact, was opposite to the prediction that would be made by the reinforcement hypothesis.

The relationship between running and events at feeding time was analyzed by timing E's entries at feeding time against event records of running in Group IF and by direct observation at feeding time. Any 